\section{Response control of responding: Discrimination and generalization of response-produced stimuli*}

\author{
REID R. VANDELL and DOUGLAS P. FERRARO \\ University of New Mexico, Albuquerque, N. Mex. 87106
}

Specific antecedent barpress-response durations emitted by rats were substituted for the exteroceptive stimuli typically used in stimulus control paradigms. The resultant response-discrimination and response-generalization paradigms produced differential rates of response along a response-duration continuum. The obtained response control over responding was analogous to the stimulus control of responding along stimulus dimensions reported in the literature. The findings were interpreted as support for the conceptualization that response control represents a special instance of stimulus control in which responding is based on response-produced stimuli.

Responses can be differentially reinforced on the basis of: (1) their correlation with an exteroceptive stimulus; (2) their correlation with an antecedent response; or (3) their qualitative or quantitative properties. Stimulus discrimination, response discrimination, and response differentiation, respectively, denote the differential responding produced by these operational paradigms.

While it is important to retain the above operational distinctions, it is useful conceptually to integrate the three paradigms by assuming that a response produces interoceptive stimuli which are isomorphic with the dimensions of the response. Under this assumption, which does not necessarily specify the identity of the hypothesized response-produced stimuli (i.e., cutaneous, kinesthetic, proprioceptive, etc.), the three operational paradigms may all be regarded as instances of stimulus discrimination. As stimulus discriminations, the three paradigms differ in the source of the stimuli (exteroceptive or interoceptive) used to define the stimulus-response correlation and thus in the degree to which the $E$ can identify or manipulate the stimuli. Discrimination Type 1 involves reinforcing responses correlated with E-produced exteroceptive stimuli; Discrimination Type 2 involves reinforcing responses correlated with interoceptive stimuli produced by antecedent responses; and Discrimination Type 3 involves reinforcing responses in the presence of interoceptive stimuli correlated with some qualitative or quantitative property of the on-going response.

The concept that responses can be

* The research was supported by Grant GB-7674 to Douglas P. Ferraro from the National Science Foundation. controlled by response-produced stimuli has a long history in psychology (cf. Goss, 1961; Grice, 1965) and has stimulated considerable research and theory. Much of the empirical literature relevant to the role of response-produced stimuli in learning has been recently reviewed in the context of several major conceptual positions (e.g., Adams, 1968; Greenwald, 1970; Taub \& Berman, 1968). It should be noted that conceptualizations which place the burden of explanation on response-produced stimuli do not necessarily lead to specification of either the properties or the identity of these stimuli. It would seem in this context that a parsimonious position to maintain is that response-produced stimuli, whatever their identity, have properties common to other (exteroceptive) stimuli. One implication of this position, and more generally of the conceptual integration of the three paradigms, is that discrimination paradigms should be demonstrable using analogous Type 2 response-discrimination paradigms Rilling \& McDiarmid, 1965) or Type 3 response-differentiation paradigms (e.g., Notterman \& Mintz, 1965).

Attempts to replicate the phenomena of stimulus control by using response control procedures in which response-produced stimuli were relevant for learning generally have been successful. A Type 3 discrimination procedure has been employed by Ferraro and his coworkers to obtain response control findings analogous to Weber's law for Type 1 discriminations $(1970)$ and to those obtained in studies of intradimensional transfer for Type 1 discriminations (1968). Additionally, generalization gradients based on antecedent responses have been phenomena obtained with Type 1 (e.g., Pliskoff \& Goldiamond, 1966; obtained with Type 2 discrimination procedures by Mechner (1958) and by Reynolds (1966). However, neither of these latter response control situations was directly analogous to Type 1 stimulus-generalization situations, since neither procedure permitted the $\mathrm{E}$ directly to manipulate the properties of the responses with respect to the dimension of generalization.

The present experiment was designed to demonstrate response control of responding along a response dimension in a manner directly analogous to stimulus control of responding along a stimulus-genéralization dimension (Kalish, 1969). More specifically, the purpose of the experiment was to obtain postdiscrimination gradients of generalization along a response duration dimension by relating response probability to a specified, E-controlled duration of an antecedent response.

The Ss were four naive male Long-Evans hooded rats, approximately 200 days old at the beginning of experimentation. The individually caged Ss were given free access to food in the home cages but were maintained at $80 \%$ of their normal body weights by restricting water intake. Ss were weighed daily and watered immediately following each experimental session at approximately the same time each day.

\section{APPARATUS}

A standard sound-attenuating operant chamber (Scientific Prototype Model A 100) was used. The response manipulanda were two small $(.6 \mathrm{~cm}$ in diam $\times 1.9 \mathrm{~cm}$ in length) levers which required approximately $15 \mathrm{~g}$ of downward force and were capable of extending and retracting (Grisham, Wolach, \& Ferraro, 1969). The two retractable levers, which were separated horizontally by $16 \mathrm{~cm}$, were located on the front panel of the chamber, $4 \mathrm{~cm}$ above the chamber floor. Located midway between the two levers was a Lehigh Valley liquid dipper which was used to present $.01 \mathrm{cc}$ of water reinforcement for $1.5 \mathrm{sec}$.

\section{PROCEDURE}

After 11 preliminary training sessions, Ss were responding reliably on a two-lever procedure in which a trial was defined as the time between successive presentations of the right lever. Each trial was initiated by the insertion of the right lever into the experimental chamber. The $S$ was required to press the right lever and hold it down for a specified minimum duration in order to meet criterion. The length of the criterion duration was increased progressively during 
preliminary training on the basis of S's performance and ranged between .15 and $4.8 \mathrm{sec}$ for Ss 1 and 2 and between 2.4 and $8 \mathrm{sec}$ for Ss 3 and 4 . A right-lever holding response which was shorter than the specified minimum holding duration was counted as an error but had no effect on the experimental contingencies. However, a criterion right-lever response duration produced an immediate retraction of the right lever and simultaneous insertion of the left lever into the experimental chamber. Responses to the left lever were then reinforced, regardless of response duration, according to a random-ratio reinforcement schedule which required an average of eight responses to produce each reinforcement. The left lever remained available for a duration of $16 \mathrm{sec}$, after which the left lever was retracted and the next trial was immediately initiated by the insertion of the right lever. Thus, each trial throughout the experiment included at least a criterion holding response on the right lever and a 16-sec presentation of the left lever. Since the timing of the criterion holding-response duration was initiated by $S$ 's leverpress response, this duration represented the minimum possible time between the insertion and retraction of the right lever. However, the actual amount of time the right lever was present on any given trial would vary as a function of S's leverpress latency and the number and/or duration of holding-response errors for that trial.

Type 2 response-discrimination training was given during the seven subsequent 189-trial experimental sessions. Under the responsediscrimination procedure, one of two right-lever holding criteria was in effect on each trial ( 0 and $2.4 \mathrm{sec}$ for Ss 1 and 2;0 and 4 sec for Ss 3 and 4 ). $A$ criterion holding response of the longer $\left(R^{D}\right)$ duration was followed, as before, by $16 \mathrm{sec}$ of reinforcement availability on the left lever. Response durations shorter than the specified criterion duration again had no effect but were counted as errors. On the other hand, a criterion holding response of $0 \mathrm{sec}\left(\mathrm{R}^{\Delta}\right)$ duration (defined simply as a leverpress) was followed by a 16-sec period of nonreinforcement on the left lever. The order of presenting the two holding criteria was randomized within blocks of nine trials, with $R^{D}$ in effect three times and $R^{\Delta}$ in effect six times within each nine-trial block.

During the last four response-discrimination training sessions, one of the six $R^{\Delta}$ trials of each nine-trial block was used to present response-generalization probe trials. In the response-generalization

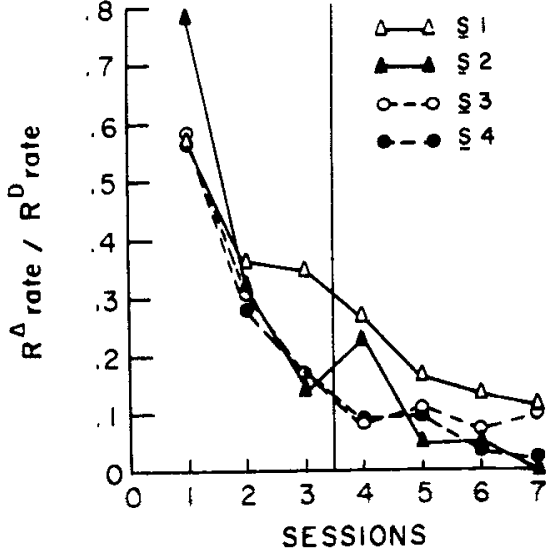

Fig. 1. Average ratio of left-lever response rate which followed a right-lever $R^{\Delta}(0 \mathrm{sec})$ to the left-lever response rate, corrected for reinforcement duration, which followed a right-lever $R^{D}(2.4 \mathrm{sec}$ for Ss 1 and $2 ; 4.0$ sec for Ss 3 and 4 ) as a function of response discrimination training sessions.

probe trials, one of seven holding criteria was in effect on the right lever. Probe trial, right-lever criterion responses were always followed by extinction on the left lever for 16 sec. The probe-trial criterion duration for Ss 1 and 2 was $0, .15, .3, .6,1.2,2.4$, or $4.8 \mathrm{sec}$ and for Ss 3 and 4 was 0 , $.25, .5,1,2,4$, or $8 \mathrm{sec}$.

Throughout the experiment, response rates on the left lever were recorded as a function of the duration of the immediately preceding criterion response emitted on the right lever.

\section{RESULTS}

The present procedure used a holding contingency which required that experimental control over the duration of the right-lever response be achieved. If complete control by the holding contingency had been obtained, no holding errors would have occurred and only one right-lever response per trial would have been emitted. Accordingly, the average number of right-lever responses per trial serves as an index of the control exerted by the holding requirement. This average, taken separately for Ss 1-4 over the seven sessions of Type 2 response-discrimination training, was, respectively, 1.33, 1.29, 1.89 , and 1.59 right-lever responses per trial. Control by the holding contingency was similarly maintained during response-generalization probe trials and was independent of the length of the generalization probe-trial criterion duration. It will be noted that the occurrence of holding-response error(s) on some trials produced trial-to-trial variation in the length of time that the right lever was present for a particular lever-holding criterion. This variation was contributed to by variability in the latency of the leverpress response to the insertion of the right lever. Thus, the length of the interval between the insertion and retraction of the right lever was not perfectly correlated with the duration of the holding-response criterion.

Regardless of the right-lever response latency or the number of right-lever responses emitted per trial, a right-lever criterion response duration always immediately preceded the presentation of the left lever. Thus, differential reinforcement of responses on the left lever was consistently correlated with the duration of the immediately antecedent right-lever response. To the extent that differential response rates were achieved on the left lever, response control (control by a Type 2 response discrimination) was demonstrated. A measure of differential responding, comparable to the often-used $S^{\Delta} / S^{D}$ stimulus discrimination ratio, was obtained by dividing the left-lever response rate which followed a right-lever $R^{\Delta}$ by the left-lever response rate, corrected for reinforcement duration, which followed a right-lever $R^{D}$. This response-discrimination ratio, $R^{\Delta} / R^{D}$, equals unity in the absence of differential responding and equals zero when a perfect response discrimination is achieved. Response discrimination ratios for the seven sessions of differential reinforcement are presented in Fig. 1. Although $R^{\Delta}$ was 0 sec for all $S s$, the value of $R^{D}$ was different between Ss 1 and $2(2.4 \mathrm{sec})$ and Ss 3 and $4(4.0 \mathrm{sec})$. Nevertheless, all $\mathrm{Ss}$ achieved a stable discrimination ratio of less than .15 by the end of training. As can be seen in Fig. 1, however, the rate and the regularity of acquiring the response discriminations were directly related to the magnitude of the difference between the $R^{D}$ and $\mathrm{R}^{\Delta}$ response durations. With the possible exception of $S 2$, differential responding after $R^{D}$ and $R^{\Delta}$ was not disrupted by the introduction of response-generalization extinction probe trials during the fourth session of discrimination training.

The left-lever response rates, which were obtained during each 16 -sec extinction period that followed the occurrence of a right-lever criterion generalization probe duration, were averaged separately for each of the seven probe response durations across the four sessions of responsegeneralization trials. The resultant response-rate generalization gradients are presented for each $\mathrm{S}$ in Fig. 2. It is apparent that response control of response rate along a response-duration dimension was 
obtained regardless of the set of duration criteria employed.

The response generalization gradients shown in Fig. 2 tended to flatten on the shorter end of the duration continuum near $R^{\Delta}=0$ and at the longest probe duration beyond $R^{D}$. However, intermediate response rates were obtained at response duration values between $R^{\Delta}$ and $R^{D}$. Comparisons between the slope values of the individual gradients are complicated by the fact that individual Ss produced different response discrimination ratios during the four sessions of generalization probe tests. It should be noted, however, that the slope of a gradient between any two response duration criteria was a function of the $R^{D}$ value and thus of the set of duration criteria employed in testing. This is most noticeable for response duration criteria in the neighborhood of 1 to 2 sec. A steep slope was obtained between 1 and 2 sec when the response discrimination $R^{D}$ was $4 \mathrm{sec}$, while a relatively flatter slope was obtained between 1.2 and 2.4 sec when the response discrimination $R^{D}$ was $2.4 \mathrm{sec}$. Thus, it would appear that differential responding along the present response dimension, indicative of response control of responding, was not absolute, but rather was dependent upon the context in which the initial response discrimination was acquired.

\section{DISCUSSION}

The usual stimulus discrimination paradigm (Type 1) involves reinforcing responses correlated with specified exteroceptive stimuli. In the present experiment, response-control paradigms were specified by replacing the exteroceptive stimuli in the stimulus-control paradigm with specific antecedent response durations emitted by S. External control of Ss' response durations was achieved by retracting the right lever after the holding criterion, timed from a leverpress response, was met. Since the insertion and retraction of the right lever produced exteroceptive stimuli, it was possible for the interval between them, and not the duration of the holding response, to serve as the temporal continuum of discrimination. While this possible confounding cannot be completely negated, the fact that trial-to-trial variations in the exteroceptively defined interval were produced by variations in leverpress latencies and in holding errors tends to render this confounding less probable. Moreover, when responding was differentially reinforced on the basis of the precise durations of antecedent responses, differential control of responding was acquired by the antecedent responses in a fashion directly analogous to that obtained in

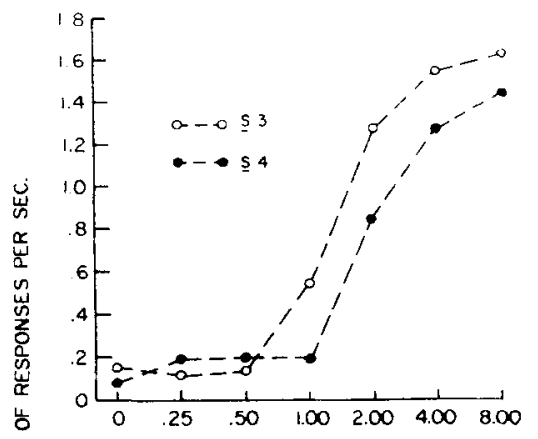

1970). The response-discrimination paradigm is probably preferable in this context to the response-differentiation paradigm because it provides a more independent means of investigating the discriminative control of behavior. In the response-differentiation paradigm, the variables which control the production of a specified on-going response may interact with those variables controlling the concurrent discrimination of that response. In the response-discrimination paradigm, the production of an antecedent response and the subsequent discrimination of that antecedent response are separate.

In summary, the bulk of the available literature clearly supports the general statement that responses do serve to control responding. This empirical generalization, in turn, supports the conceptualization that response discrimination and response differentiation may be subsumed as special instances of stimulus discrimination, the stimuli in the latter case being exteroceptive and, in the former cases, being produced by antecedent or on-going responses. In the present instance, the obtained response discrimination and response generalization would be conceptualized as special instances of stimulus discrimination and stimulus generalization in which the properties of the interoceptive stimuli produced by antecedent response durations served as the stimulus dimension of discrimination and generalization. It will be noted that this conceptual integration does not necessarily require the acceptance of a unitary process underlying the three discrimination paradigms. To the contrary, it is possible that related, but nonparallel, processes may be necessary to explain the discontinuities sometimes produced by the different paradigms (e.g., Reynolds, 1966).

\section{REFERENCES}

ADAMS, J. A. Response feedback and learning. Psychological Bulletin, 1968, 70, 486-504.

FERRARO, D. P., \& GRILLY, D. M. Response differentiation: A psychophysical method for response produced stimuli. Perception \& Psychophysics, $1970,7,206-208$.

FERRARO, D. P., GRILLY, D. M., \& TANG, R. S. W. Transfer of a differentiation along a response continuum. Journal of Comparative \& Physiological Psychology, 1968, 66. 793-796.

GOSS. A. E. Early behaviorism and verbal mediating responses. American Psychologist, $1961,16,285-298$.

GREENWALD, A. G. Sensory feedback mechanisms in performance control: With special reference to the ideo-motor mechanism. Psychological Review. 1970. $77,73-97$.

prior response-differentiation findings that phenomena analogous to stimulus control phenomena are obtainable with response-control procedures (Ferraro et al, 1968; Ferraro \& Grilly,
GRICE, $G$. R. Do responses evoke responses? American Psychologist, 1965 , $20,282-294$.

GRISHAMI. M. G., WOLLACH, A. H.. \& 
FERRARO, D. P. An inexpensive retraction mechanism. Behavior Research Methods and Instrumentation, 1969, 1, 233.

KALISH, H. I. Stimulus generalization. In M. Marx (Ed.), Learning: Processes. Toronto: Macmillan, 1969. Pp. 207-297.

MECHNER, F. Probability relations within response sequences maintained under ratio reinforcement. Journal of the Experimenta' Analysis of Behavios, 1958 ,
1. $109-121$.

NOTTERMAN, J. M., \& MINTZ, E. Dynamics of response. New York: Wiley, 1965

PLISKOFF, S. S., \& GOLDIAMOND, I Some discriminative properties of fixed ratio performance in the pigeon. Journal of the Experimental Analysis of Behavior. $1966,9,1-9$

REYNOLDS, G. S. Discrimination and emission of temporal intervals by pigeons.
Journal of the Experimental Analysis of Behavior, 1966, 9,65-68.

RILLING, M \& McDIARMID, C. Signal detection in fixed ratio schedules. Science, 1965, 148,526-527.

TAUB,E, \& BERMAN, A. J. Movement and learning in the absence of sensory feedback. In S. J. Freedman (Ed.), The neuropsychology of spatially oriented behavior. Homewood, Ill: Darsey Press, 1968 . 